\title{
Noninvasive Home Mechanical Ventilation in Adult Myotonic Dystrophy Type 1: A Systematic Review
}

\author{
Bettine A. H. Vosse ${ }^{a}$ Charlotte Seijger ${ }^{b} \quad$ Nicolle Cobben ${ }^{a}$ Baziel van Engelen ${ }^{c}$ \\ Sander M. J. van Kuijk ${ }^{d}$ Catharina Faber ${ }^{e}$ Peter Wijkstra ${ }^{b}$ \\ aDepartment of Pulmonary Diseases and Home Mechanical Ventilation, Maastricht University Medical Center+, \\ Maastricht, The Netherlands; bepartment of Pulmonary Diseases and Home Mechanical Ventilation, University of \\ Groningen, University Medical Center Groningen, Groningen, The Netherlands; 'Department of Neurology, Donders \\ Institute for Brain, Cognition and Behaviour, Radboud University Medical Center, Radboud University Nijmegen, \\ Nijmegen, The Netherlands; ${ }^{d}$ Department of Clinical Epidemiology and Medical Technology Assessment, Maastricht \\ University Medical Center+, Maastricht, The Netherlands; e Department of Neurology, School of Mental Health and \\ Neuroscience, Maastricht University Medical Center+, Maastricht, The Netherlands
}

\section{Keywords}

Myotonic dystrophy $\cdot$ Noninvasive ventilation $\cdot$ Respiratory insufficiency · Respiration · Artificial · Ventilators ·

Mechanical

\begin{abstract}
Introduction: Chronic hypercapnic respiratory failure induces considerable morbidity and mortality in patients with myotonic dystrophy type 1 (DM1). This study systematically reviews the effects of noninvasive home mechanical ventilation (HMV) on gas exchange, quality of life, survival, and compliance in DM1 patients. Methods: A systematic Medline and Embase search was performed (January 1995 to January 2020). Records were screened for eligibility criteria, data were extracted from included studies, and risk of bias was assessed. We present findings mainly using a narrative synthesis. Results: Twenty-eight relevant full-text articles were screened for eligibility criteria. Nine studies were included. Randomized controlled trials were not found. Studies had either an observational $(n=8)$ or interventional $(n=1)$ design. In the pooled data analysis, HMV showed to improve mean oxygen saturation with $4.8 \%$ and decreased mean car-
\end{abstract}

karger@karger.com www.karger.com/res

Karger"

BOPEN ACCESS
(C) 2021 The Author(s)

Published by S. Karger AG, Basel

This is an Open Access article licensed under the Creative Commons Attribution-NonCommercial-4.0 International License (CC BY-NC) (http://www.karger.com/Services/OpenAccessLicense), applicable to the online version of the article only. Usage and distribution for commercial purposes requires written permission. bon dioxide values with $3 \mathrm{~mm} \mathrm{Hg}$. Compliance varied widely between studies, from no use to more than $12 \mathrm{~h}$ per day. Quality of life was not studied extensively, but some studies reported positive effects of HMV on symptoms of chronic respiratory failure. HMV may improve survival in DM1 patients with chronic hypercapnic respiratory failure. Conclusion: This review shows that HMV can improve gas exchange and relieve symptoms with a possible survival benefit in DM1 patients with chronic hypercapnic respiratory failure. Future studies should focus on developing strategies to optimize the timing of HMV initiation and to promote compliance.

(c) 2021 The Author(s)

Published by S. Karger AG, Basel

\section{Introduction}

Myotonic dystrophy type 1 (DM1) is the most frequently inherited type of adult-onset muscular dystrophy, with a reported prevalence of $0.5-18.1$ per 100,000 person years and

B.A.H. Vosse and C. Seijger equally contributed to this paper.
Correspondence to:

Bettine A.H. Vosse, bettine.vosse @ mumc.nl 
Table 1. Study eligibility criteria

\begin{tabular}{ll}
\hline Criterion & Eligibility criteria \\
\hline Population & Studies in myotonic dystrophy type 1 patients with chronic hypercapnic respiratory failure \\
\hline Intervention & Studies involving long-term home mechanical ventilation, using noninvasive positive pressure ventilation \\
\hline Comparator & Studies with comparator and noncomparator designs \\
\hline Outcome & Studies reporting on gas exchange, quality of life, survival, and compliance with HMV \\
\hline Study design & $\begin{array}{l}\text { Empirical quantitative and qualitative studies published in English in the past } 25 \mathrm{yr}^{*}, \text { excluding case reports, } \\
\text { conference reports, studies without original research, and studies in children }<18 \mathrm{yr}\end{array}$ \\
\hline
\end{tabular}

HMV, home mechanical ventilation. * The review was restricted to studies published in the past 25 years to ensure that findings are relevant to current practice.

an estimated prevalence as high as 1 per 2,500 [1,2]. Patients have a reduced life expectancy of 54-60 years [3,4]. Respiratory failure is the primary cause of death in DM1, which is thought to result from a complex mechanism with important contribution of respiratory muscle weakness, but also a reduced central respiratory drive, decreased chest wall compliance and upper airway obstruction [4-11]. These factors result in reduced lung volumes, sputum stasis, and atelectasis causing considerable morbidity due to increased risks of pneumonia and respiratory failure. Invasive or noninvasive home mechanical ventilation (HMV) can be used in case of chronic hypercapnic respiratory failure. Benefits of mainly noninvasive HMV on gas exchange, symptoms and survival have been described [12-14]. However, in daily clinical practice, treatment of DM1 patients with HMV is often complex due to lack of improvement of symptoms and treatment intolerance which consequently leads to a reduced compliance to therapy in comparison with other neuromuscular disorders $[12,15,16]$. Therefore, HMV in DM1 patients can be perceived as a burdensome treatment for patients, their family, and healthcare professionals. The aim of this systematic review was to evaluate the effect of HMV on gas exchange, quality of life (QoL), survival, and compliance to therapy in adult DM1 patients with chronic hypercapnic respiratory failure in order to improve daily clinical practice and provide future directions for further research.

\section{Methods}

Protocol

This review protocol was registered in PROSPERO (International Prospective Register of Systematic Reviews, www.crd.york. ac.uk/prospero; registration number CRD42020168349) and was conducted in accordance with PRISMA reporting standards [17].

\section{Search Strategy}

With the help of a clinical librarian, 2 authors (C.S. and B.V.) performed a comprehensive literature search in MEDLINE and Embase databases (from January 1995 to January 2020). The main search terms were "myotonic dystrophy," "respiratory insufficiency," and "artificial respiration." Different synonyms, medical subheadings, and Boolean pooling operators were used. The reproducible search strategy is included in online supplementary Appendix 1 (for all online supplementary material, see www. karger.com/doi/10.1159/000515453). Searches were performed in title and abstract and limited to studies in adult humans being presented in the English language. Supplementary searching techniques included hand-searching of included studies' reference lists.

\section{Study Selection}

Citations retrieved from electronic database searches were uploaded to Covidence (www.covidence.org). Titles and abstracts were independently screened by 2 reviewers (C.S. and B.V.) using eligibility criteria; a third reviewer resolved any uncertainties (P.W.). The absolute level of agreement between the 2 reviewers regarding study inclusion was $>95 \%$. Then, full texts were obtained and selection was performed based on predefined eligibility criteria shown in Table 1.

\section{Study Outcomes}

The primary outcome of this study was the effect of HMV on gas exchange (especially oxygen and carbon dioxide), QoL, and survival in DM1 patients. Secondary outcome parameter was the compliance to HMV and factors that influence compliance.

\section{Data Collection Process}

The following data were extracted independently by 2 reviewers (C.S. and B.V.), using a standardized extraction form consisting of title, authors, publication year, study design, sample size, sample characteristics (mean age, and gender), available forced vital capacity (FVC), and muscular impairment rating scale (MIRS), which was specifically developed for DM1 [18], HMV indication, and information about outcome measurements (gas exchange, QoL, survival, and compliance). 
Fig. 1. PRISMA flow chart illustrating the study selection process. PRISMA, Preferred Reporting Items for Systematic Reviews and Meta-Analyses.

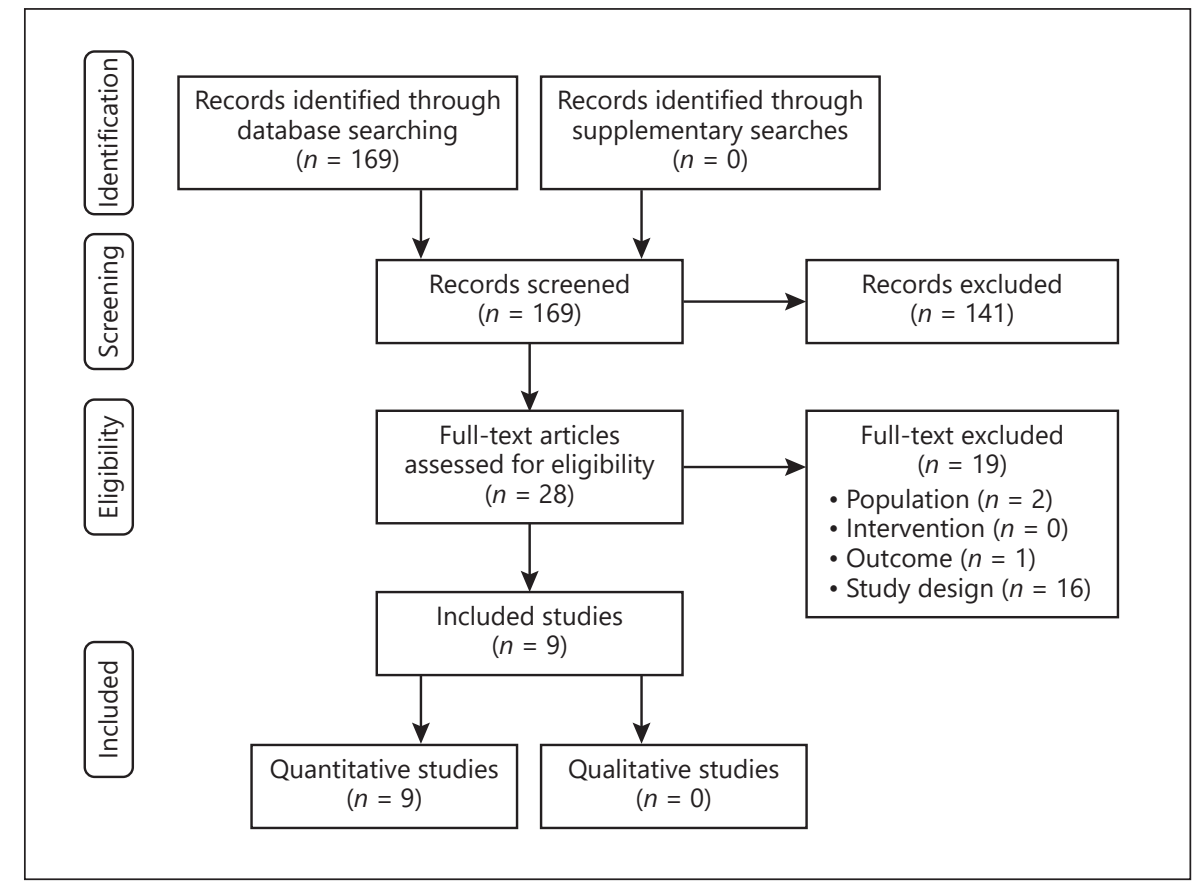

Quality Assessment

The risk of bias was assessed using the Newcastle-Ottawa Scale (NOS) designed for nonrandomized studies [19]. Two reviewers (C.S. and B.V.) independently scored the studies by their quality of patient selection, comparability, and outcome on a nine-star scoring system. Discrepancies were discussed and resolved. Due to significant between-study heterogeneity, risk of bias was not assessed across the cumulative evidence.

Data Synthesis and Statistical Analysis

In case multiple studies reported on the same outcome parameter(s) and reported both baseline and follow-up values, we performed a meta-analysis. As the methods of the included studies were heterogeneous, we used the random-effects model to account for between-study heterogeneity. The I-squared statistic was used to estimate the percentage of variation in results due to between-study heterogeneity. In case a meta-analysis was not possible, we used a narrative synthesis method. We brought together studies examining similar processes or reporting similar outcomes, identified where data agreed and where it conflicted, and provided an indication of the volume and quality of the evidence.

\section{Results}

\section{Study Selection}

After removing duplicates, the literature search resulted in 169 records (shown in Fig. 1). Following title and abstract screening, 28 were considered for full-text evaluation. Of these, we included 9 articles based on full-text.
Reasons for exclusion were design (16), outcome (1), and patient population (2).

\section{Study Characteristics}

An overview of included studies and main outcomes is presented in Table 2. No randomized controlled trials were found. All studies had a quantitative design. One interventional study was found and all other studies were of observational design (retrospective or prospective). The 9 included studies were performed in 6 different countries. Two studies were performed by Boussaïd et al. $[14,15]$ who used, upon enquiry, mainly the same cohort for both studies. In total, $841 \mathrm{DM} 1$ patients were included, of which 385 started HMV, with variable age, disease severity (based on the MIRS, FVC, and triplet repeat length; available details in Table 2), and different indications for HMV (daytime hypercapnia, nocturnal hypoventilation, sleepdisordered breathing, or restrictive pulmonary function with a vital capacity ( $\mathrm{VC})<50 \%$ of predicted).

\section{Quality Assessment}

The quality of the individual studies is shown in Table 2 . The mean quality score (i.e., the mean number of stars awarded according to the NOS) of the studies was 4.8 (range 3-7). The comparability item, which scores a maximum of 2 stars, could only be assessed in 1 study. The level of agreement between the 2 reviewers was $>93 \%$ and, after discussion, disagreements were resolved. 


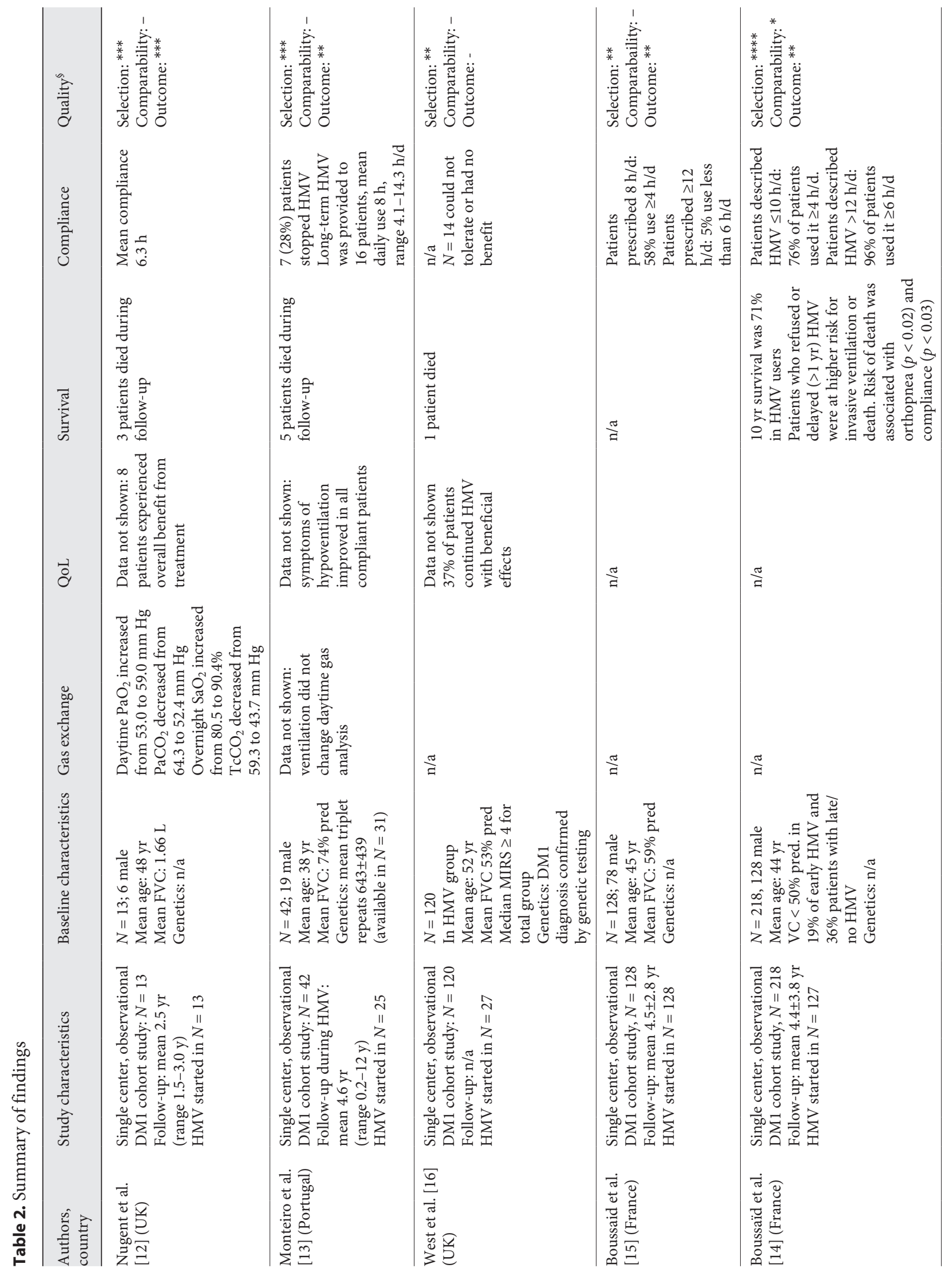




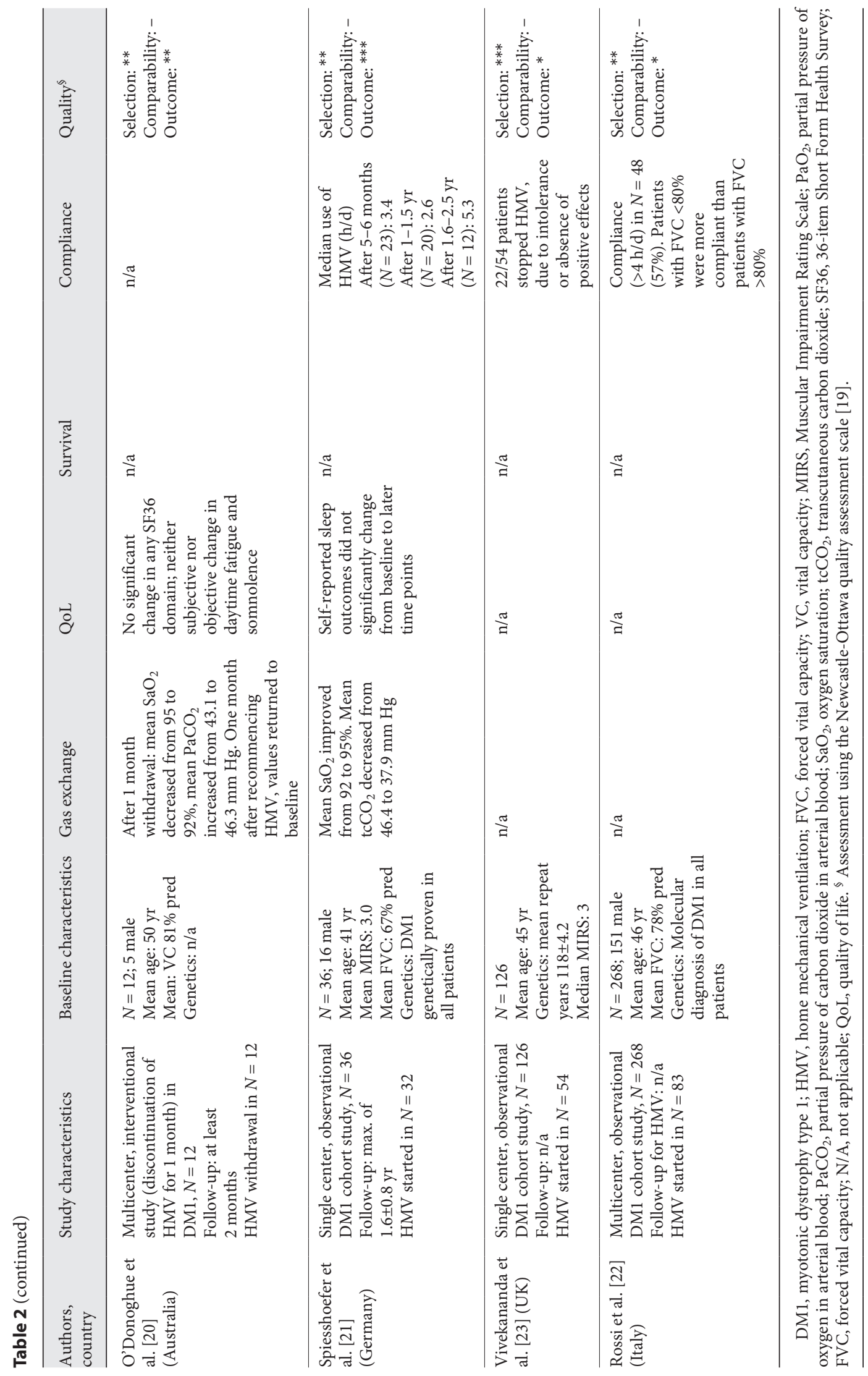


Fig. 2. Forest plots: pooled data on gas exchange. Change in $\mathrm{PCO}_{2}$ after 1 month on $\mathrm{HMV}$ (a); change in $\mathrm{PO}_{2}$ after 1 month on $\mathrm{HMV}$ (b); change in $\mathrm{SaO}_{2}$ after 1 month on $\mathrm{HMV}$ (c). $\mathrm{PCO}_{2}$, partial pressure of carbon dioxide in blood; $\mathrm{PO}_{2}$, partial pressure of oxygen in blood; $\mathrm{SaO}_{2}$, oxygen saturation; $\mathrm{HMV}$, home mechanical ventilation.

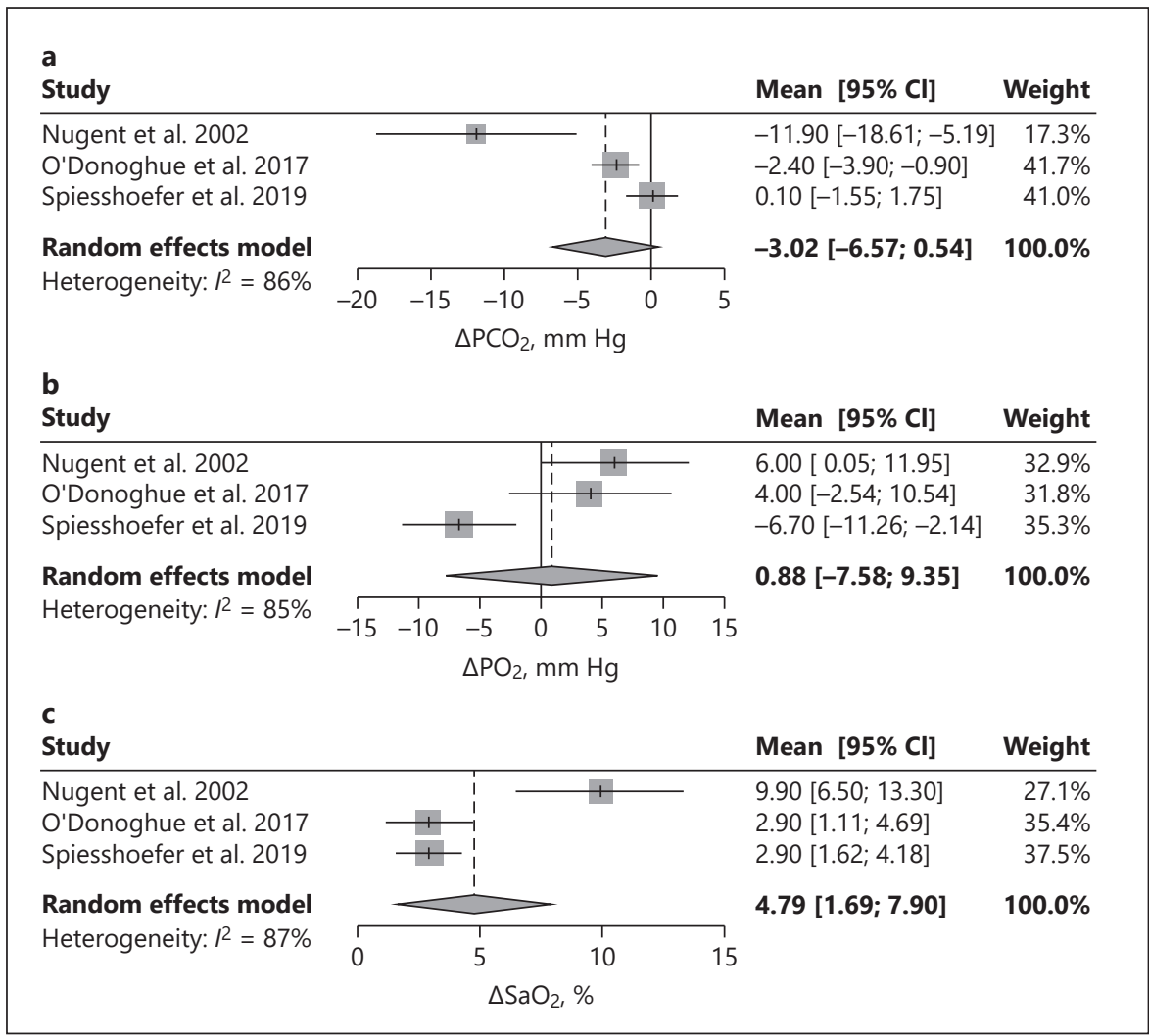

\section{Gas Exchange}

Four studies reported the effects of HMV on gas exchange $[12,13,20,21]$. After 1 night on HMV, oxygenation and transcutaneous carbon dioxide significantly improved in 32 patients, showing that the mean transcutaneous carbon dioxide normalized from $46.4 \pm 5.3$ to $39.5 \pm 5.2 \mathrm{~mm} \mathrm{Hg}$ and the mean oxygen saturation $\left(\mathrm{SaO}_{2}\right)$ improved from 92 to $95 \%$ [21]. These results were found to be long lasting for more than 2 years. In addition, gas exchange improved more in patients with higher treatment compliance [21]. A smaller study of 13 patients also showed improvement in gas exchange using HMV, without reaching normocapnia [12]. Another small study describes that daytime gas analysis did not change in 25 patients, but baseline and outcome data were not presented [13]. A prospective pilot study of O'Donoghue et al. [20] found that elective withdrawal of long term HMV during 1 month in 12 patients with chronic hypercapnic respiratory failure (mean diurnal partial pressure of carbon dioxide in arterial blood $\left[\mathrm{PaCO}_{2}\right]<45 \mathrm{~mm} \mathrm{Hg}$ on HMV) resulted in a decrease of $\mathrm{SaO}_{2}$ and an increase of $\mathrm{PaCO}_{2}$, while both values returned to baseline after starting HMV again. The pooled results of 2 retrospective observational studies and 1 prospective study showed an overall positive effect on partial pressure of carbon dioxide in blood $\left(\mathrm{PCO}_{2}\right)$, partial pressure of oxygen in blood $\left(\mathrm{PO}_{2}\right)$, and $\mathrm{SaO}_{2}$ as shown in Figure $2[12,20,21]$. After approximately 1 month of $\mathrm{HMV}, \mathrm{PCO}_{2}$ decreased while $\mathrm{PO}_{2}$ and $\mathrm{SaO}_{2}$ increased. $\mathrm{PCO}_{2}$ and $\mathrm{PO}_{2}$ were measured in arterial blood gases in 2 studies $[12,20]$ and in capillary blood gases in the third study [21].

\section{Quality of Life}

The effects of HMV on QoL were only investigated in the withdrawal study of $\mathrm{O}^{\prime}$ Donoghue et al. [20] No significant changes were found in any 36-item Short Form Health Survey domain between ' 1 month withdrawal of HMV' and after ' 1 month reintroduction', although nonsignificant changes ( $p$-values 0.11-0.18), favoring HMV, were found for the physical functioning, role limitation due to physical problems, social functioning, and vitality domains [20]. In 3 studies the effects of HMV on symptoms were described $[12,13,16]$. Results were variable, with the smallest effects described by West et al. [16] who found that $37 \%$ patients with respiratory failure using 
HMV continued it with beneficial effects. Nugent et al. [12] described that 8 of 10 patients subjectively experienced an overall benefit from treatment with less daytime sleepiness and improved nocturnal sleep. The study of Monteiro et al. [13] confirmed the findings that symptoms of chronic respiratory failure improved in compliant patients. The effects of HMV on sleep were investigated in 2 studies [20,21]. Positive effects were only found regarding the apnea-hypopnea index and oxygen desaturation index [21]. Both studies did not find clinically relevant differences for neither subjective nor objective sleep parameters $[20,21]$.

\section{Compliance}

Six studies described the compliance to HMV [12-15, $21,22]$. These were difficult to summarize due to differences in definitions and a lack of information about cessation reasons. Mean hours of HMV use ranged from $3.4 \mathrm{~h} /$ day to $8 \mathrm{~h} /$ day. In $57-76 \%$ of patients, HMV was used for more than $4 \mathrm{~h} /$ day. The percentage of patients who did not use or stopped HMV varied between 20 and $54 \%$. One prospective study of $128 \mathrm{DM} 1$ patients investigated the compliance with HMV and cessation predictors [15]. They demonstrated that compliance during the first year was $25 \%$ higher when symptoms of respiratory failure were initially present, whereas initiation during acute respiratory failure reduced the relative compliance by $29 \%$. Long-term compliance data showed that $42 \%$ of patients who were prescribed $8 \mathrm{~h} \mathrm{HMV} /$ day used it for less than $4 \mathrm{~h}$ /day and $28 \%$ did not use it at all. From the patients who were prescribed $\geq 12 \mathrm{~h} /$ day, $5 \%$ used it less than $6 \mathrm{~h} /$ day. Long-term compliance was positively associated with symptoms of respiratory failure $(+52 \%, p<$ $0.01)$ and nocturnal arterial oxygen desaturation $(+23 \%$, $p<0.01)$. Risk of HMV cessation was associated with excessive leaks (HR $=7.81$, IC [1.47-41.88], $p<0.01$ ), dysfunctions that required emergency technical interventions $(\mathrm{HR}=12.58$, IC [1.22-129.69], $p<0.03)$, and an increase of body mass index (BMI), especially BMI $\geq 30.0$ $(\mathrm{HR}=33.8$ IC [2.63-433.7]. Risk of HMV cessation was lower in patients with a professional occupation or who were undergoing professional training ( $\mathrm{HR}=0.11 \mathrm{IC}$ [0.02-0.77]) [15]. Other frequently described reasons for stopping treatment were intolerance to $\mathrm{HMV}$, absence of positive effects, uncomfortable masks, and excessive noises of the device $[16,23]$. Eighteen percent of longterm HMV users experienced problems (which are not significantly related to HMV cessation), such as excessive leaks, skin or mucosa irritation, and gastric inflation [15].

\section{Survival}

One of the first studies published regarding HMV in DM1 suggested a survival benefit in their cohort of patients receiving assisted ventilation $(n=13)$. Three patients died (one of them was not compliant), while the rest received long-term assisted ventilation for a mean period of 27 months (range 2-76) [12]. Other studies describe mortality in their cohort using different periods of followup $[13,16]$. Most evidence for an association between HMV and mortality is derived from a large prospective cohort study conducted in France which compared survival in patients who accepted HMV promptly with those who refused or delayed HMV. Of the 127 patients who used HMV, the 10 -year mortality was $29 \%$. In the whole group, during a follow-up period of 959 patient-years, 53 patients died with an annual rate of 53/959 ( 1 per 6 person-years). Mean age at death was $58.2 \pm 8.7$ years. Patients died due to respiratory causes (45\%), cardiac causes $(6 \%)$, cancer (4\%), and unknown causes (45\%). The risk of a severe event (defined as invasive mechanical ventilation or death) was significantly higher in the group of patients who started HMV later or not at all as compared to the group who started HMV within 1 year after meeting the criteria to start HMV. In the HMV group, orthopnea and poor compliance to the HMV were independently and significantly associated with death (respectively, HR $2.37,95 \%$ CI $1.17-4.8, p<0.02$ and HR 3.26, 95\% CI $1.32-$ $8.04, p<0.03)[14]$.

\section{Discussion}

This review shows that the current evidence for HMV in DM1 patients is mainly based on clinical expertise and a small number of observational studies. Nevertheless, these studies provide important information and enable us to determine future directions with regard to research questions aiming to optimize HMV care in DM1.

Gas exchange was positively influenced by HMV in DM1 patients, at daytime as well as overnight $[12,20,21]$, which is similar to other neuromuscular and chest wall disorders [24]. In general, the efficacy of HMV is based on 2 rationales. First, it may rest the fatigued respiratory muscles, thereby increasing strength and endurance, which may improve pulmonary mechanics by increasing chest wall compliance and lung volumes. Second, it leads to an increased ventilatory response to carbon dioxide $\left(\mathrm{CO}_{2}\right)$, which is probably the main mechanism for longterm improvement of daytime gas exchange in patients treated with HMV [25-27]. However, hypercapnia in 
DM1 patients is sometimes disproportionate to the respiratory muscle weakness, which suggests a central cause of $\mathrm{CO}_{2}$ insensitivity and may explain why DM1 patients tend to experience less HMV treatment benefits [7, 11].

With regard to indication for HMV initiation in DM1 patients, consensus meetings are mainly based on expert opinion in combination with low evidence studies and have suggested to start HMV when there are symptoms suggestive of chronic respiratory insufficiency in combination with daytime or nocturnal hypercapnia or FVC $<50 \%$ of predicted $[28,29]$. However, certain symptoms, like excessive daytime sleepiness and fatigue may be classified as signs of hypoventilation, but in DM1 patients, they are likely of multifactorial origin and, therefore, the result of HMV on such symptoms may be disappointing and discouraging for patients and caregivers [30, 31]. Also, patients with preserved respiratory muscle strength and hypercapnia may respond differently and may, therefore, need another approach with regard to (timing of the initiation of) HMV.

Data about the effects of HMV on QoL and symptoms in DM1 are limited. QoL was investigated in only 1 pilot study and remained unchanged after elective withdrawal of HMV and restarted 1 month later [20]. Beneficial effects on symptoms are described, although this was only descriptively studied in a small subset of patients $[12,13$, 16]. Regarding sleep aspects, the apnea-hypopnea index improved, but HMV did not change the sleep efficiency and sleep architecture [20, 21]. In other diseases, HMV improved QoL [32], mainly assessed by the Severe Respiratory Insufficiency questionnaire and the Maugeri Respiratory Failure questionnaire $[33,34]$. The $S^{3}$-NIV questionnaire, a new and easy to use short questionnaire is validated to measure the experience of HMV users [35].

Compliance to HMV ranged from no use at all to 2-12 $\mathrm{h} /$ day $[12,13,15,16]$. Better compliance resulted in more improvement of symptoms, whereas poor compliance is associated with higher mortality $[14,23]$. One of the predictors of noncompliance was HMV initiation during acute respiratory failure. It is questionable whether those patients have indeed chronic respiratory failure. Do they actually have the right indication for chronic HMV? Patients might recover from an acute event and become noncompliant because of the absence of indication for HMV. Another reason could be that these patients are not well informed in advance about the burden and the intended aim of treatment. Increase of BMI is another worrisome predictor for noncompliance, as overweight is present in $50 \%$ of DM1 patients which further decreases lung volumes and, therefore, probably accelerates the de- velopment of respiratory failure [36,37]. Compliance was found to be better in patients with symptoms of respiratory failure at time of initiation, and patients having a professional occupation or training had a lower risk of NIV cessation [15]. To our knowledge, the impact of intelligence, cognition, and affective symptoms such as apathy has never been studied in relation to compliance to HMV, while we know that intellectual impairment and apathy are very common in DM1 patients $[38,39]$. In our clinical experience, these factors might obstruct therapy due to difficulties in clarifying its importance and negatively influencing the motivation to continue.

Survival is markedly reduced in DM1 patients with respiratory failure as the primary cause of death (50\%) next to cardiac causes (30\%) [3]. Only 1 large study on HMV and survival has been published and found a significantly higher risk of dying in patients who refused to start HMV or started later than in patients with an early HMV initiation [14]. However, this result could also be due to baseline differences with a lower $\mathrm{VC}$, which is a known independent risk factor for mortality and a higher $\mathrm{PaCO}_{2}$ in the patients who refused or started HMV later [40]. Therefore, survival benefits of HMV in DM1 patients are still questionable.

\section{Future Directions}

In general, studies with DM1 patients are complex, mainly because DM1 is a very heterogeneous disorder with variable involvement of multiple organs, resulting in severe impairment in some patients, and only mild symptoms in others. Since HMV in DM1 patients has been offered for more than 20 years, it is considered unethical to perform a randomized controlled trial to answer questions about optimal compliance and treatment effectiveness. Based on the presented benefits, we recommend that every DM1 patient is subjected to a basic respiratory evaluation (spirometry and preferably blood gas analysis) in order to screen for the need of HMV. For future HMV studies in this population, more knowledge should be gained about which patients benefit the most of HMV, what is the optimal timing of initiating HMV, and what is the required minimal compliance to HMV. In order to achieve this optimal personalized HMV therapy, the effects of education, supportive follow up, as well as the role of social support (including a bed partner) will be meaningful topics for future research.

Extensive specific patient- and therapy-related items should be collected. Specific patient-related factors need to include respiratory and neurological functioning, as well as assessment of QoL and symptoms. Socio-econom- 
ic status and care dependency are additional factors. It is preferred to use disease-specific questionnaires, such as the DM1 Activity and Participation Scale to assess daily functioning and the Rasch-built Fatigue and Daytime Sleepiness Scale to assess fatigue and sleepiness [41, 42]. Therapy-related factors such as ventilator settings and interfaces used should be taken into account. Survival studies should correct for independent predictors for mortality in DM1, such as diabetes, need for walking support, cardiac measurements, and VC [40]. Recently, the multicenter study Differential Response to Noninvasive Ventilation in Myotonic Dystrophy was started in the Netherlands (www.trialregister.nl; registration number NL7972) with the aim to profile a multidimensional response to noninvasive HMV and identify patients with DM1 that do (or do not) respond well to NIV.

\section{Conclusion}

Noninvasive HMV can improve gas exchange and relieve symptoms with a possible survival benefit in DM1 patients with chronic hypercapnic respiratory failure. It is recommendable that every DM1 patient is subjected to a respiratory assessment. Compliance to HMV is variable and influences the effect of treatment. In order to achieve an optimal personalized HMV treatment, future studies should focus on developing strategies which enable optimization of the timing of HMV initiation and promotion of compliance.

\section{Statement of Ethics}

The reviewed studies comply with the guidelines for human studies and were conducted ethically in accordance with the World Medical Association Declaration of Helsinki.

\section{Conflict of Interest Statement}

The authors have no conflicts of interest to declare.

\section{Funding Sources}

No funding was received.

\section{Author Contributions}

All authors contributed to the study concept and design. B.V. and C.S. performed the study search, study selection, and wrote the manuscript. S.v.K. performed statistical analyses. Outcomes were interpreted by all authors. All authors contributed to the critical review of the manuscript for important intellectual content.

\section{References}

1 Theadom A, Rodrigues M, Roxburgh R, Balalla S, Higgins C, Bhattacharjee R, et al. Prevalence of muscular dystrophies: a systematic literature review. Neuroepidemiology. 2014;43(3-4):259-68.

2 Johnson NE. Myotonic muscular dystrophies. Continuum. 2019;25(6):1682-95.

3 Groh WJ, Groh MR, Shen C, Monckton DG, Bodkin CL, Pascuzzi RM. Survival and CTG repeat expansion in adults with myotonic dystrophy type 1 . Muscle Nerve. 2011;43(5): 648-51.

4 de Die-Smulders CE, Höweler CJ, Thijs C, Mirandolle JF, Anten HB, Smeets HJ, et al. Age and causes of death in adult-onset myotonic dystrophy. Brain. 1998;121(Pt 8):155763.

5 Groh WJ, Groh MR, Saha C, Kincaid JC, Simmons Z, Ciafaloni E, et al. Electrocardiographic abnormalities and sudden death in myotonic dystrophy type 1 . N Engl J Med. 2008;358(25):2688-97.

6 Mathieu J, Allard P, Potvin L, Prévost C, Bégin P. A 10-year study of mortality in a cohort of patients with myotonic dystrophy. Neurology. 1999;52(8):1658-62.
7 Begin P, Mathieu J, Almirall J, Grassino A. Relationship between chronic hypercapnia and inspiratory-muscle weakness in myotonic dystrophy. Am J Respir Crit Care Med. 1997; 156:133-9.

8 Kilburn KH, Eagan JT, Sieker HO, Heyman A. Cardiopulmonary insufficiency i myotonic and progressive muscular dystrophy. $\mathrm{N}$ Engl J Med. 1959;261:1089-96.

9 Begin R, Bureau MA, Lupien L, Bernier JP, Lemieux B. Pathogenesis of respiratory insufficiency in myotonic dystrophy: the mechanical factors. Am Rev Respir Dis. 1982;125: 312-8.

10 Carroll JE, Zwillich CW, Weil JV. Ventilatory response in myotonic dystrophy. Neurology. 1977;27(12):1125-8.

11 Poussel M, Thil C, Kaminsky P, Mercy M, Gomez E, Chaouat A, et al. Lack of correlation between the ventilatory response to $\mathrm{CO} 2$ and lung function impairment in myotonic dystrophy patients: evidence for a dysregulation at central level. Neuromuscul Disord. 2015; 25(5):403-8.
12 Nugent AM, Smith IE, Shneerson JM. Domiciliary-assisted ventilation in patients with myotonic dystrophy. Chest. 2002;121(2): 459-64.

13 Monteiro R, Bento J, Gonçalves MR, Pinto T, Winck JC. Genetics correlates with lung function and nocturnal ventilation in myotonic dystrophy. Sleep Breath. 2013;17(3):1087-92.

14 Boussaid G, Prigent H, Laforet P, Raphael JC, Annane D, Orlikowski D, et al. Effect and impact of mechanical ventilation in myotonic dystrophy type 1: a prospective cohort study. Thorax. 2018.

15 Boussaid G, Lofaso F, Santos DB, Vaugier I, Pottier S, Prigent H, et al. Factors influencing compliance with non-invasive ventilation at long-term in patients with myotonic dystrophy type 1: a prospective cohort. Neuromuscul Disord. 2016;26:666-74.

16 West SD, Lochmüller H, Hughes J, Atalaia A Marini-Bettolo C, Baudouin SV, et al. Sleepiness and sleep-related breathing disorders in myotonic dystrophy and responses to treatment: a prospective cohort study. J Neuromuscul Dis. 2016;3(4):529-37. 
17 Shamseer L, Moher D, Clarke M, Ghersi D, Liberati A, Petticrew M, et al. Preferred reporting items for systematic review and meta-analysis protocols (PRISMA-P) 2015: elaboration and explanation. BMJ. 2015;350: g7647.

18 Mathieu J, Boivin H, Meunier D, Gaudreault M, Bégin P. Assessment of a disease-specific muscular impairment rating scale in myotonic dystrophy. Neurology. 2001;56(3):336-40.

19 Wells GA, Shea B, O'Connell D, Peterson J, Welch V, Losos M, Tugwell P. The NewcastleOttawa Scale (NOS) for assessing the quality of nonrandomised studies in meta-analyses. Assessed in June, 2020.

20 O’Donoghue FJ, Borel JC, Dauvilliers Y, Levy P, Tamisier R, Pépin JL. Effects of 1-month withdrawal of ventilatory support in hypercapnic myotonic dystrophy type 1 . Respirology. 2017;22(7):1416-22.

21 Spiesshoefer J, Runte M, Heidbreder A, Dreher M, Young P, Brix T, et al. Sleep-disordered breathing and effects of non-invasive ventilation on objective sleep and nocturnal respiration in patients with myotonic dystrophy type I. Neuromuscul Disord. 2019;29(4): 302-9.

22 Rossi S, Della Marca G, Ricci M, Perna A, Nicoletti TF, Brunetti V, et al. Prevalence and predictor factors of respiratory impairment in a large cohort of patients with Myotonic Dystrophy type 1 (DM1): a retrospective, cross sectional study. J Neurol Sci. 2019;399:118-24.

23 Vivekananda U, Turner C. A model to predict ventilator requirement in myotonic dystrophy type 1. Muscle Nerve. 2019;59(6):683-7.

24 Annane D, Chevrolet JC, Chevret S, Raphael JC. Nocturnal mechanical ventilation for chronic hypoventilation in patients with neuromuscular and chest wall disorders. Cochrane Database Syst Rev. 2000(2): CD001941.

25 Annane D, Quera-Salva MA, Lofaso F, Vercken JB, Lesieur O, Fromageot C, et al.
Mechanisms underlying effects of nocturnal ventilation on daytime blood gases in neuromuscular diseases. Eur Respir J. 1999;13(1): 157-62.

26 Nickol AH, Hart N, Hopkinson NS, Moxham J, Simonds A, Polkey MI. Mechanisms of improvement of respiratory failure in patients with restrictive thoracic disease treated with non-invasive ventilation. Thorax. 2005;60(9): 754-60.

27 Hill NS. Noninvasive ventilation. Does it work, for whom, and how?. Am Rev Respir Dis. 1993;147(4):1050-5.

28 Boentert M, Cao M, Mass D, De Mattia E, Falcier E, Goncalves M, et al. Consensus-based care recommendations for pulmonologists treating adults with myotonic dystrophy type 1. Respiration. 2020;99(4):360-8.

29 Sansone VA, Gagnon C. participants of the 207th EW: 207th ENMC Workshop on chronic respiratory insufficiency in myotonic dystrophies: management and implications for research, 27-29 June 2014, Naarden, The Netherlands. Neuromuscul Disord. 2015;25: $432-42$.

30 Laberge L, Gagnon C, Dauvilliers Y. Daytime sleepiness and myotonic dystrophy. Curr Neurol Neurosci Rep. 2013;13(4):340.

31 Laberge L, Dauvilliers Y, Bégin P, Richer L, Jean S, Mathieu J. Fatigue and daytime sleepiness in patients with myotonic dystrophy type 1: to lump or split? Neuromuscul Disord. 2009;19(6):397-402.

32 MacIntyre EJ, Asadi L, McKim DA, Bagshaw SM. Clinical outcomes associated with home mechanical ventilation: a systematic review. Can Respir J. 2016;2016:6547180.

33 Windisch W, Freidel K, Schucher B, Baumann $\mathrm{H}$, Wiebel M, Matthys $\mathrm{H}$, et al. The severe respiratory insufficiency (SRI) questionnaire: a specific measure of health-related quality of life in patients receiving home mechanical ventilation. J Clin Epidemiol. 2003; 56(8):752-9.
34 Carone M, Bertolotti G, Anchisi F, Zotti AM, Donner CF, Jones PW. Analysis of factors that characterize health impairment in patients with chronic respiratory failure. Quality of life in chronic respiratory failure group. Eur Respir J. 1999;13(6):1293-300.

35 Dupuis-Lozeron E, Gex G, Pasquina P, Bridevaux PO, Borel JC, Soccal PM, et al. Development and validation of a simple tool for the assessment of home noninvasive ventilation: the S(3)-NIV questionnaire. Eur Respir J. 2018;52:1801182.

36 Seijger CG, Drost G, Posma JM, van Engelen BG, Heijdra YF. Overweight is an independent risk factor for reduced lung volumes in myotonic dystrophy type 1 . PLoS One. 2016; 11(3):e0152344.

37 Gagnon C, Chouinard MC, Laberge L, Brisson D, Gaudet D, Lavoie M, et al. Prevalence of lifestyle risk factors in myotonic dystrophy type 1. Can J Neurol Sci. 2013;40(1):42-7.

38 van der Velden BG, Okkersen K, Kessels RP, Groenewoud J, van Engelen B, Knoop H, et al. Affective symptoms and apathy in myotonic dystrophy type 1 a systematic review and meta-analysis. J Affect Disord. 2019;250:260-9.

39 Jean S, Richer L, Laberge L, Mathieu J. Comparisons of intellectual capacities between mild and classic adult-onset phenotypes of myotonic dystrophy type 1 (DM1). Orphanet J Rare Dis. 2014;9:186.

40 Wahbi K, Porcher R, Laforêt P, Fayssoil A, Bécane HM, Lazarus A, et al. Development and validation of a new scoring system to predict survival in patients with myotonic dystrophy type 1. JAMA Neurol. 2018;75(5):573-81.

41 Symonds T, Randall JA, Campbell P. Review of patient-reported outcome measures for use in myotonic dystrophy type 1 patients. Muscle Nerve. 2017;56(1):86-92.

42 Hermans MC, Merkies IS, Laberge L, Blom EW, Tennant A, Faber CG. Fatigue and daytime sleepiness scale in myotonic dystrophy type 1. Muscle Nerve. 2013;47(1):89-95. 